\title{
Individual differences in neonatal white matter are associated with executive function at 3 years of age
}

\author{
Sarah J. Short ${ }^{1,4} \cdot$ Michael T. Willoughby $^{2} \cdot$ Marie Camerota $^{3} \cdot$ Rebecca L. Stephens $^{4} \cdot$ Rachel J. Steiner $^{4}$. \\ Martin Styner ${ }^{4,5}$. John H. Gilmore ${ }^{4}$
}

Received: 11 February 2019 / Accepted: 3 September 2019 / Published online: 13 September 2019

○) Springer-Verlag GmbH Germany, part of Springer Nature 2019

\begin{abstract}
The development of executive function (EF) in early childhood contributes to social and academic aspects of school readiness and facilitates emerging self-regulatory competence. Numerous efforts are underway to identify aspects of early brain development that contribute to emerging EF. Existing research supports the importance of multiple white matter tracts for EF in older children and adults. However, this research has not been extended to young children. In this study, we consider neonatal white matter microstructure in relation to children's performance on a battery of EF tasks three years later. We obtained diffusion tensor imaging data from a sample of neonates $(N=27)$ shortly after birth. At 3 years of age, children completed a computerized battery of EF tasks. The primary data analyses involved regression models estimated for each white matter tract. Multiple demographic and measure-related covariates were included in each model. A follow-up analysis of tracts determined to be associated with EF examined individual data points along those fibers. Among the white matter tracts analyzed, the cingulum was significantly associated with EF at 3 years of age. Specifically, lower axial diffusivity values along the cingulum were associated with increased performance on the EF battery. Results are discussed as providing initial evidence that individual differences in neonatal brain structure may facilitate the acquisition of EF abilities in early childhood. These findings are consistent with previous research that supports the value of the cingulum for higher-order cognitive abilities. Cautions and implications of these results are considered.
\end{abstract}

Keywords Infant brain development · Diffusion tensor imaging · Executive function · Early childhood · White matter · Cognitive development

Electronic supplementary material The online version of this article (https://doi.org/10.1007/s00429-019-01955-0) contains supplementary material, which is available to authorized users.

Sarah J. Short

sjshort@wisc.edu

1 Center for Healthy Minds and the Department of Educational Psychology, University of Wisconsin, $625 \mathrm{~W}$. Washington Ave., Madison, WI 53703, USA

2 RTI International, Research Triangle Park, USA

3 Center for Developmental Science, University of North Carolina at Chapel Hill, Chapel Hill, USA

4 Department of Psychiatry, University of North Carolina at Chapel Hill, Chapel Hill, USA

5 Department of Computer Science, University of North Carolina at Chapel Hill, Chapel Hill, USA

\section{Introduction}

Executive function (EF) is a construct that encompasses cognitive processes supporting goal-directed behaviors and problem-solving skills that are critical for numerous functions of daily life and for learning in general. Individual differences in EF contribute to multiple aspects of children's social and academic competency (Diamond 2013). The predictive value of early EF in terms of later academic achievement is also well established (e.g., Blair and Diamond 2008). However, efforts to identify the biological and behavioral precursors of emerging EF during the first three years of life have only recently begun (Hendry et al. 2016). Most of this work has only considered how specific aspects of infant cognition (e.g., attention, processing speed) relate to emerging EF. Here, we consider the contributions of neonatal brain structure as a complementary approach. Recent methodological developments in magnetic resonance imaging (MRI) 
have enabled scientists to characterize brain structure and function in the infant brain (Geng et al. 2012; Gilmore et al. 2010; Knickmeyer et al. 2008). The first few years of life represent one of the most rapid and prolific periods of growth in the human brain, during which cerebral volume reaches approximately $80 \%$ of adult form (Knickmeyer et al. 2008). Concurrently, during this age range, children demonstrate significant improvement in a variety of behaviors and cognitive skills. Behavioral correlates of gray matter have been researched in depth; however, less is understood about links between white matter properties and cognition (Fields 2010; Filley and Fields 2016; Zatorre et al. 2012).

White matter is critical for behavior and cognition as it allows for efficient information processing and rapid communication between cortical regions. Although there is a high degree of variability across white matter tracts at birth in terms of myelination (Geng et al. 2012; Lee et al. 2017), all major tracts are established by the end of normal gestation. In fact, whole brain connectivity analyses have revealed that the structural network organization in neonates closely resembles the adult brain (Ball et al. 2014; Keunen et al. 2017; Yap et al. 2011). There is substantial evidence supporting the association between neuroimaging measures of white matter microstructure and cognition in adults (Bendlin et al. 2010; Kerchner et al. 2012) and older children (Nagy et al. 2004; Peters et al. 2014). However, very little is known about such brain-behavior relationships during early childhood.

Diffusion tensor imaging (DTI) techniques provide indirect measures of white matter tract microstructure via the directional diffusion of water molecules. The reconstructed diffusion tensor image captures properties of white matter microstructure that are commonly quantified via tensor derived scalar parameters including fractional anisotropy (FA), radial diffusivity (RD), and axial diffusivity (AD) (for a review see Beaulieu 2002). FA is a composite of the eigenvalues that comprise RD and $\mathrm{AD}$. As such, FA values are sensitive to multiple aspects of white matter microstructure. In general, FA values increase with age while the other diffusion parameters typically decrease across childhood and into early adulthood, reflecting increased maturation, myelination, and efficiency (Lebel et al. 2008; Rasmussen et al. 2017; Seghete et al. 2013). RD is derived from the diffusion of water molecules perpendicular to white matter fiber bundles and is thus thought to reflect information about axon myelination as well as other microstructural tissue properties. AD is derived from the parallel diffusion of water molecules along white matter fiber bundles and is thought to reflect information about fiber organization (Beaulieu 2002). Interpretation of directional information provided from $\mathrm{RD}$ and $\mathrm{AD}$ is best represented when the direction of fibers aligns, such as in the corpus callosum or the internal capsule. When considered in relation to cognition, RD has been useful for characterizing brain-behavior relations during later stages of life, when the brain is fully myelinated. Although they are not yet fully myelinated, the majority of white matter fiber bundles are in place at birth (Dubois et al. 2014; Ouyang et al. 2019). As such, AD is thought to be the most stable measure in neonates for examining associations with later cognitive function (Lee et al. 2017; Rasmussen et al. 2017). Thus, the analyses in this study focus on AD, but also include analysis of diffusion parameters FA and RD that provide general and complementary information.

Little is known about the early structural neural antecedents that subserve critical behavioral and cognitive functions such as EF (Baum et al. 2016; Gao et al. 2009). In older children and adults, neuroimaging studies using DTI have identified several white matter tracts connecting frontal, parietal, temporal, and subcortical structures as important for supporting performance on EF tasks. Specifically, white matter microstructure of the cingulum (Kantarci et al. 2011; Bettcher et al. 2016), the superior longitudinal fasciculus (SLF) (Burzynska et al. 2011; Perry et al. 2009; Sasson et al. 2013), the arcuate fasciculus (Lebel et al. 2013), and the uncinate (Perry et al. 2009; Sasson et al. 2013) have most consistently been linked with EF in adolescent and adult samples. In children, research on the white matter correlates of EF has mostly focused on atypical or clinical samples. However, the few studies linking EF and white matter microstructure in typically developing children support a similar set of tracts to those found in adolescent and adult samples. For example, in a large sample that included children as young as 8 years the microstructural properties of the cingulum were related to EF performance (Peters et al. 2014). Other studies of children and adolescents have shown the microstructure of the SLF to be correlated with specific aspects of EF, such as set shifting (Urger et al. 2015) and working memory (Vestergaard et al. 2011). While very little research of this sort has been conducted with infants, associations with working memory and microstructure of the arcuate fasciculus have been identified in 1-year-old infants (Short et al. 2013) and another recent study has provided evidence linking the microstructural properties of the uncinate at 6 months of age with later joint attention behaviors at 9 months of age (Elison et al. 2013). Joint attention has strong established associations with emerging EF (Miller and Marcovitch 2015; Vaughan Van Hecke et al. 2011), with a number of studies suggesting overlapping neurobiology (Mundy and Jarrold 2010; Mundy and Newell 2007).

Investigating whether infant white matter microstructure is reflective of later emerging foundational cognitive skills such as EF is important for supporting healthful developmental trajectories in children. Similarly, conducting this research during sensitive periods of neural development may provide a critical means of identifying children at risk for neurodevelopmental and learning disorders, which would 
be invaluable as these earliest stages of life often represent the greatest opportunities for preventive interventions. In this study, we examine the microstructure of white matter tracts that are most often found to relate to EF in older populations to determine whether the structural integrity of these tracts just after birth is associated with children's later performance on direct assessments of EF tasks at 3 years of age. To the best of our knowledge, no other studies have examined these associations so early in development. Thus, this study represents the first attempt to relate individual differences in neonatal brain structure to emergent EF in young children.

\section{Methods and materials}

\section{Participants}

The current study sample was made up of a subset of typically developing infants $(N=27)$ from ongoing longitudinal studies examining early brain development (Gilmore et al. 2010; Knickmeyer et al. 2008, 2016). Pregnant women were recruited from outpatient OB-GYN clinics at two university medical centers. Healthy infants were included in the current study if they had a quality DTI scan at birth (with no major abnormalities), had turned 3 years old at the time of EF data collection, and had successfully completed at least two EF tasks.

Demographic information is summarized in Table 1. Mothers completed an average of 16 years of education (SD 3.3; range 10-22 years), roughly equivalent to a 4-year college degree, and primarily identified as White (70\%). There was a slightly higher number of male children participating $(n=17)$ than females. Infants were born with an average gestational age of 262.7 days (SD 19.3; range 226-285 days). At the time of the neonatal DTI scan, infants were, on average, 29 days old (SD 19 days; range 9-79 days). The range in age at time of scan reflects an effort to account for differences in development due to gestational age at birth. To roughly equate the length of time since conception for all infants, scan acquisition was delayed for children born before a full-term length. Therefore, infants born earlier were scanned at a slightly older chronological age. At the time of EF testing, children were an average of 3.4 years old (SD 0.2; range 3.1-3.8 years).

Written informed consent was obtained from the parents of all infant participants at the time of recruitment to the original study and again prior to participating in the EF assessments. The Institutional Review Boards of the University of North Carolina School of Medicine and Duke University Medical Center approved this study.

Table 1 Sample description

\begin{tabular}{|c|c|c|c|}
\hline & $N$ & $\mathrm{M}(\mathrm{SD})$ & Range \\
\hline Child gestational age at birth (days) & 27 & $262.7(19.3)$ & $226-285$ \\
\hline Child chronological age at scan (days) & 27 & $29(19)$ & $9-79$ \\
\hline Child age at EF testing (years) & 27 & $3.4(0.2)$ & $3.1-3.8$ \\
\hline Maternal education (years) & 27 & $16.1(3.3)$ & $10.0-22.0$ \\
\hline Bubbles reaction time (ms) & 27 & $1377(353)$ & $869-2739$ \\
\hline Number EF tasks completed & 27 & $4.26(1.40)$ & $2-6$ \\
\hline Arrows (mean correct) & 22 & $0.41(0.27)$ & $0.00-1.00$ \\
\hline Silly sounds stroop (mean correct) & 16 & $0.46(0.24)$ & $0.06-0.94$ \\
\hline Animal go/no-go (mean correct) & 9 & $0.81(0.24)$ & $0.25-1.00$ \\
\hline Working memory span (mean correct) & 22 & $0.31(0.17)$ & $0.06-0.72$ \\
\hline Pick the PICTURE (mean correct) & 21 & $0.54(0.14)$ & $0.19-0.72$ \\
\hline Something's the same (mean correct) & 25 & $0.62(0.11)$ & $0.37-0.83$ \\
\hline \multirow[t]{2}{*}{ EF composite (mean correct) } & 27 & $0.42(0.15)$ & $0.17-0.77$ \\
\hline & & & $N(\%)$ \\
\hline Child sex (male) & & & $17(63 \%)$ \\
\hline Maternal race (White) & & & $19(70 \%)$ \\
\hline Maternal race (African American) & & & $7(26 \%)$ \\
\hline Maternal race (Native American) & & & $1(4 \%)$ \\
\hline Maternal ethnicity (Hispanic) & & & $1(4 \%)$ \\
\hline
\end{tabular}

$M$ mean, $S D$ standard deviation 


\section{Executive function touch (EF touch)}

EF touch is a computerized battery of EF tasks developed for use with children aged 3-6 years that was initially created, administered, and studied in paper-and-pencil (i.e., flipbook) formats (Willoughby and Blair 2011; Willoughby et al. 2012, 2013). The computerization of tasks improved the efficiency, standardization, and sensitivity of the previous battery of tasks (Willoughby and Blair 2016). The EF Touch program runs in a Windows OS environment. A capacitive touch-screen monitor records children's responses. Abbreviated descriptions for the tasks that are used in this study appear in the supplemental materials. Briefly, we administered three inhibitory control tasks (variations of go/no-go, stroop, and flanker tasks), two working memory tasks (variations of self-ordered pointing and span tasks), and a single attention shifting task. In addition to these six tasks of components of EF, one simple reaction time (SRT) task was administered via the EF Touch program, which required children to respond to stimuli on the screen as fast as they could. Full task descriptions for EF and SRT tasks appear in the supplemental materials.

The mean performance across the six individual EF tasks was used to create an overall EF composite score. The merits of using a composite to improve the reliability and interpretability of individual differences in EF abilities have been established elsewhere (Willoughby et al. 2016). Consistent with our previous work involving 3-year-old children (Willoughby et al. 2010), some children were unable to complete all of the tasks. As such, the number of tasks completed was included as a covariate in models involving the prediction of the EF composite score. Additionally, we calculated children's SRT by averaging together their mean response time across all valid trials in the SRT task. This was also included as a covariate in all models.

\section{Image acquisition}

MRI data were acquired on a 3 T Siemens Allegra $(n=15)$ or Tim Trio scanner (Siemens Medical Systems) with the following sequencing parameters for both scanners: 42 directions of diffusion sensitization were acquired at a $b$-value of $1000 \mathrm{~s} / \mathrm{mm}^{2}$, in addition to seven images with no diffusion weighting for reference. The parameters were as follows: TR/TE/Flip angle $=7680 / 82 / 90^{\circ}$, slice thickness $=2 \mathrm{~mm}$, and in-plane resolution $=2 \times 2 \mathrm{~mm}$, with a total of 60-72 slices.

\section{Diffusion tensor imaging analysis}

A study-specific quality control protocol was applied to all raw diffusion-weighted image (DWI) data using DTIPrep (http://www.nitrc.org/projects/dtiprep), which included automated slice-wise and gradient-wise artifact detection, as well as eddy current and motion correction (Liu et al. 2010; Oguz et al. 2014). This was followed by visual inspection of all DWIs to exclude additional gradients containing artifacts not detected during the automatic processing. Out of 42 gradients, there were, on average, 3.96 excluded from the images (SD 5.08), with a majority of scans only having 2 or fewer gradients removed. On average, 5.93 gradients were excluded from the Allegra DWIs (SD 5.99), and 1.50 gradients were excluded from the Trio DWIs (SD 1.83). Though the number of gradients excluded was significantly different between scanners $[t(25)=2.46$, $p<0.05]$, this was not related to any of the other study variables (i.e., DTI, EF, or covariates). Skull and nonbrain tissue were masked out using the Brain Extraction Tool (BET; Smith 2002) and corrected manually if necessary. Diffusion tensors were computed using weighted least squares fitting (Goodlett et al. 2009). Eigenvalues $(\lambda 1 \geq \lambda 2 \geq \lambda 3$ ) and corresponding eigenvectors were calculated to obtain the diffusion properties (AD, FA, RD).

Shortly after birth, the neonatal brain is largely unmyelinated and thus the diffusion anisotropy is low, which can hinder tracing of fiber tracts in their entirety when performed on an individual scan. To overcome this issue, participant data were registered to a neonate-specific atlas that was built from a large sample of typically developing neonates (for more information, see https://www.nitrc .org/projects/uncebds_neodti and http://www.nitrc.org/ projects/dtiatlasbuilder; Verde et al. 2014). For the current analyses, bilateral white matter tracts were selected a priori: arcuate, superior longitudinal, and uncinate fasciculi and cingulum. DTI fiber profiles were then generated for these a priori selected white matter tracts and data from individual participants were obtained by sampling the fiber tracts in the individual DTI image using the atlas mapping information (refer to Fig 1 below and supplemental materials for tract descriptions).

Quality control measures for all DTI data sets were used for each of the following steps of the image processing pipeline: initial image collection, subject registration to the atlas, fiber tractography, and data generation along each fiber tract. Participant DTI images were visually and quantitatively checked for successful mapping into the atlas space. The mapped FA images were first checked for gross deformation errors using MRIWatcher (https:// www.nitrc.org/projects/mriwatcher/). Then, regional tensor similarity measures for six major white matter regions were evaluated as described by Wang et al. (2011). Finally, tract profile quality control (Goodlett et al. 2009) was performed via correlational analysis for each participant in relation to the atlas profile. 
Fig. 1 White matter fiber tracts selected a priori to be associated with emergent executive function are depicted in an infant glass brain. Uncinate = blue; superior longitudinal fasciculus = green; arcuate fasciculus $=$ red; cingulum $=$ yellow

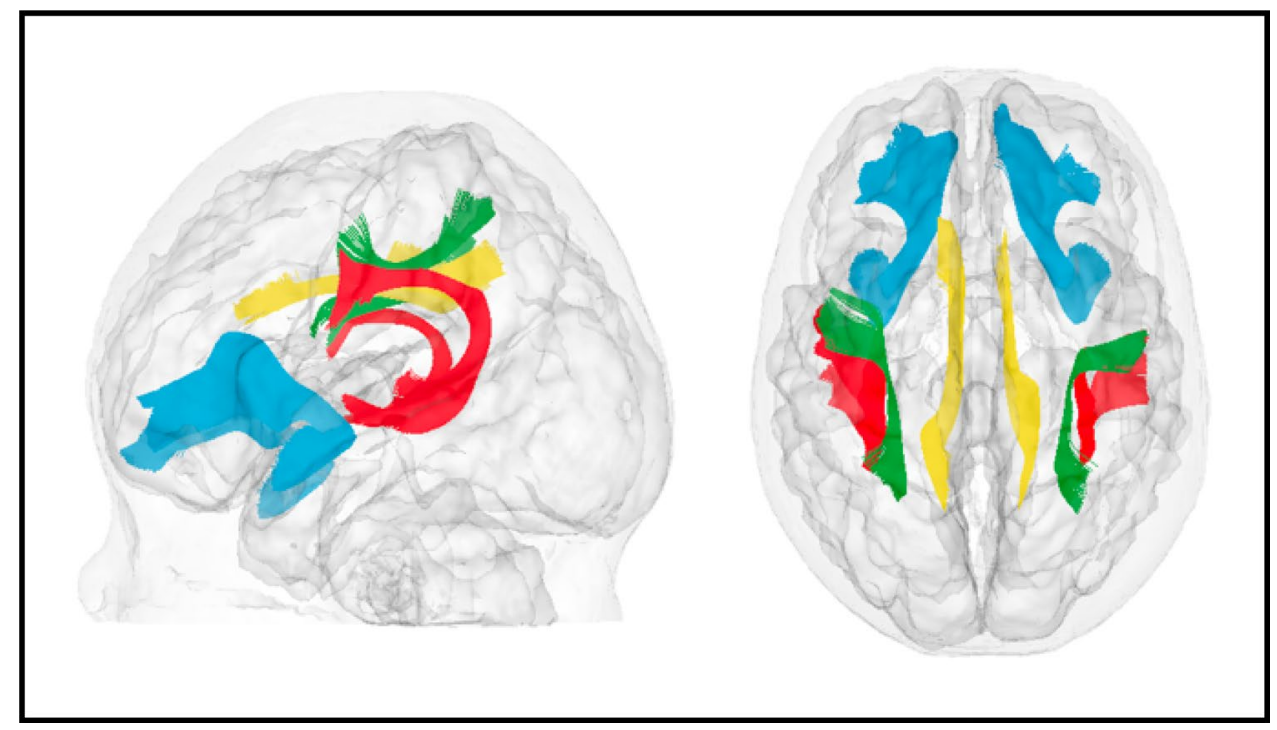

\section{White matter tract profile extraction}

White matter tracts generated from the atlas image were resampled within the DTI images of each participant. DTIAtlasFiberAnalyzer was used to define a curvilinear reparameterization of the fiber tracts (Verde et al. 2014). DTI property profiles for $\mathrm{AD}, \mathrm{FA}$, and RD were extracted for each participant along each tract. Diffusion property profiles for AD, FA, and RD were extracted at evenly spaced points along each fiber tract, and these profiles were averaged across entire tracts to generate tract average values used in the analyses of the current study. In a secondary analysis, we included individual data points along the entire white matter tract in a functional regression analysis described in the analytic strategy below.

\section{Analytic strategy}

Analyses proceeded in multiple phases: preliminary analyses included bivariate correlations between individual white matter tracts (focusing on $\mathrm{AD}$ values) from the neonatal scan, demographic variables, and the EF composite score at 3 years of age. We observed strong inter-correlations between respective tract average values ( $r$ 's ranging from 0.57 to 0.96 , all $p<0.01$ ). As such, in subsequent analyses, each tract was considered separately as a predictor of the EF composite to avoid problems with multicollinearity. Next, a series of ordinary least squares regression models were estimated in which the EF composite was regressed separately on each tract while accounting for covariates. Child gestational age, chronological age at time of scan, and gender, maternal education, scanner type, simple reaction time, and the number of EF tasks completed were included as covariates because they were correlated with either focal predictors (white matter tract averages) or EF outcomes both in this study and in previous research (e.g., Davis et al. 2011; Rasmussen et al. 2017). Graphical methods were used throughout to check model assumptions (including the impact of highly influential cases). Finally, to confirm and further probe significant findings from regression analyses of tract averages, we used Functional Analysis of Diffusion Tensor Tract Statistics (FADTTS) to evaluate curves of diffusion properties along white matter tracts in relation to children's EF scores, while accounting for the aforementioned covariates (Zhu et al. 2011). Tracts that were significantly associated with EF at the global level were followed up with FADTTS local analyses of all AD values along the white matter tract to identify specific regional associations. Benjamini-Hochberg false discovery rate (FDR) was used to correct for multiple comparisons. All primary analyses were conducted using SAS version 9.3. FADTTS analyses were conducted in Matlab.

\section{Results}

\section{Sample description}

Summary statistics for child and primary caregiver demographic information and child EF task performance are reported in Table 1. Children successfully completed (i.e., passed training items) an average of 4.3 of the 6 possible EF tasks and correctly answered between $31 \%$ and $81 \%$ of items on individual EF tasks. The mean EF composite score was $42 \%$ correct (SD 15; range 17-77\% correct). Age at testing was not significantly associated with children's EF performance or number of tasks completed. 


\section{Bivariate associations}

Bivariate correlations among AD values for each white matter tract, the EF composite, and covariates were first examined to verify the inclusion of each covariate of non-interest in the primary regression analyses. Covariates included demographic factors (child gender, child chronological age at scan, scanner type, gestational age at birth, and maternal education), simple reaction time, and the number of EF tasks completed. Results of correlations among the EF composite and covariates are summarized in Table 2. The EF composite was significantly associated with the number of tasks completed and maternal education and was moderately related to gender. There were no significant associations between the EF composite and gestational age at birth or chronological age at scan (all $p>0.10$; see Table 2). There were multiple significant associations between $\mathrm{AD}$ values and covariates (see Table 3). Similar patterns were found for FA and RD in relation to covariates (see Supplemental Tables 1,2).

\section{Regression models}

Regression models were estimated separately for each white matter tract. A synopsis of the model fit statistics along with the corresponding standardized regression coefficients for the unique contributions of white matter tracts appears in Table 4. The cingulum tracts (both left and right) were significantly related to the EF composite, although only the right cingulum survived Benjamini-Hochberg FDR correction. Each one-standard deviation unit decrease in right cingulum $\mathrm{AD}$ values was associated with a 0.83 standard deviation unit increase in performance on the EF composite $(\beta=-0.83,95 \%$ CI $[-1.18$ to -0.47$])$. The model-implied associations
Table 2 Bivariate correlations between executive function performance and covariates
Table 3 Bivariate correlations between executive function, covariates, and neonatal DTI (axial diffusivity) tract averages

\begin{tabular}{lllllllll}
\hline & EF & Mat. Ed & GA & CA & SRT & Gender $^{\dagger}$ & Scanner & Task Comp. \\
\hline EF & - & & & & & & & \\
Mat. Ed & $0.40^{*}$ & - & & & & & \\
GA & 0.29 & $0.35^{+}$ & - & & & & \\
CA & -0.12 & -0.17 & $-0.68^{* *}$ & - & & & \\
SRT & -0.09 & -0.06 & 0.20 & -0.09 & - & & \\
Gender $^{\dagger}$ & $0.38^{+}$ & 0.11 & 0.18 & 0.02 & -0.05 & - & \\
Scanner & -0.05 & -0.05 & 0.12 & 0.11 & 0.00 & -0.07 & - & \\
Task Comp. & $0.69^{* *}$ & $0.39^{*}$ & $0.63^{* *}$ & $-0.48^{*}$ & -0.12 & 0.30 & -0.06 & -
\end{tabular}

$E F$ executive function, $G A$ gestational age at birth, $C A$ chronological age at scan, $S R T$ simple reaction time, Mat. Ed. maternal education, Task Comp. number of EF tasks completed

${ }^{+} p<0.10, * p<0.05, * * p<0.01$. ${ }^{\dagger}$ In all analyses, gender was coded as $0=$ female and $1=$ male

\begin{tabular}{lllllrrrl}
\hline & EF & Mat. Ed & GA & CA & SRT & Gender $^{\dagger}$ & Scanner & Task Comp. \\
\hline CGC-L & $-0.40^{*}$ & -0.25 & -0.13 & $-0.41^{*}$ & 0.04 & -0.07 & -0.06 & -0.17 \\
CGC-R & $-0.56^{* *}$ & $-0.59^{* *}$ & -0.12 & -0.29 & 0.27 & 0.08 & -0.27 & -0.20 \\
UNC-L & -0.17 & -0.28 & $-0.33^{+}$ & $-0.40^{*}$ & 0.00 & 0.14 & -0.27 & -0.19 \\
UNC-R & -0.26 & -0.30 & $-0.40^{*}$ & -0.29 & -0.03 & 0.08 & $-0.36^{+}$ & -0.23 \\
ARC-L & $-0.40^{*}$ & -0.22 & -0.30 & -0.24 & 0.31 & 0.25 & -0.20 & $-0.38^{*}$ \\
ARC-R & -0.28 & -0.24 & -0.31 & -0.26 & 0.18 & 0.22 & -0.29 & -0.28 \\
SLF-L & $-0.35^{+}$ & -0.27 & $-0.36^{+}$ & -0.20 & 0.25 & 0.18 & -0.11 & -0.29 \\
SLF-R & -0.21 & $-0.38^{+}$ & $-0.36^{+}$ & -0.25 & 0.14 & 0.16 & -0.18 & -0.24 \\
\hline
\end{tabular}

$E F$ executive function, $G A$ gestational age at birth, $C A$ chronological age at MRI scan, $S R T$ simple reaction time, Mat. Ed. maternal education, Task Comp. number of EF tasks completed, $C G$ cingulum, UNC uncinate Fasciculus, $A R C$ arcuate fasciculus, $S L F$ superior longitudinal fasciculus

${ }^{+} p<0.10,{ }^{*} p<0.05, * * p<0.01$. ${ }^{\dagger}$ In all analyses, gender was coded as $0=$ female and $1=$ male 
Table 4 Models predicting age 3-year executive function from neonatal DTI tracts (axial diffusivity)

\begin{tabular}{|c|c|c|c|c|c|c|c|c|c|c|c|c|}
\hline \multirow[t]{2}{*}{ Predictors } & \multicolumn{12}{|c|}{$\begin{array}{l}\text { Average EF } \\
\beta\end{array}$} \\
\hline & & Left & Right & & Left & Right & & Left & Right & & Left & Right \\
\hline DTI tract & CGC & $-0.54 *$ & $-0.83 * * *$ & UNC & 0.30 & -0.23 & ARC & -0.21 & -0.04 & SLF & -0.30 & 0.08 \\
\hline GA & & $-0.63 *$ & $-0.49 *$ & & 0.11 & -0.48 & & -0.40 & -0.27 & & -0.55 & -0.15 \\
\hline CA & & -0.43 & $-0.34^{+}$ & & 0.50 & -0.09 & & -0.04 & 0.11 & & -0.13 & 0.22 \\
\hline Gender $^{\dagger}$ & & $-0.33 *$ & $-0.22^{+}$ & & -0.13 & -0.19 & & -0.16 & -0.16 & & -0.17 & -0.15 \\
\hline Scanner & & 0.11 & -0.13 & & 0.03 & 0.00 & & 0.03 & 0.02 & & 0.07 & 0.02 \\
\hline Mat. Ed. & & 0.13 & $-0.28^{+}$ & & 0.21 & 0.16 & & 0.19 & 0.18 & & 0.17 & 0.20 \\
\hline SRT & & 0.12 & $0.28 *$ & & 0.05 & 0.10 & & 0.16 & 0.09 & & 0.19 & 0.06 \\
\hline Task Comp. & & $0.66^{* *}$ & $0.74 * * *$ & & $0.81 * *$ & $0.79 * *$ & & $0.75 * *$ & $0.80 * *$ & & $0.80 * *$ & $0.80^{* *}$ \\
\hline$F(8,18)$ & & $5.34 * *$ & $10.72 * * *$ & & $3.47 *$ & $3.44 *$ & & $3.50 *$ & $3.31 *$ & & $3.80 * *$ & $3.33 *$ \\
\hline Adjusted $R^{2}$ & & 0.57 & 0.75 & & 0.43 & 0.43 & & 0.43 & 0.42 & & 0.46 & 0.42 \\
\hline
\end{tabular}

Value in italics represents the coefficient that remained significant after correction for multiple comparisons

$E F$ executive function, $G A$ gestational age at birth, $C A$ chronological age at scan, Mat. Ed. maternal education, SRT simple reaction time, Task Comp. number of EF tasks completed, $C G C$ cingulum, $U N C$ uncinate fasciculus, $A R C$ arcuate fasciculus, $S L F$ superior longitudinal fasciculus ${ }^{+} p<0.10, * p<0.05, * * p<0.01, * * * p<0.001$. ${ }^{\dagger}$ In all analyses, gender was coded as $0=$ female and $1=$ male

Fig. 2 Neonatal differences in axial diffusivity $(\mathrm{AD})$ of the left and right cingulum tracts were negatively associated with children's performance on an executive function (EF) battery at 3 years of age. Plot includes predicted values controlling for all covariates. After FDR correction, only the right cingulum remained significant

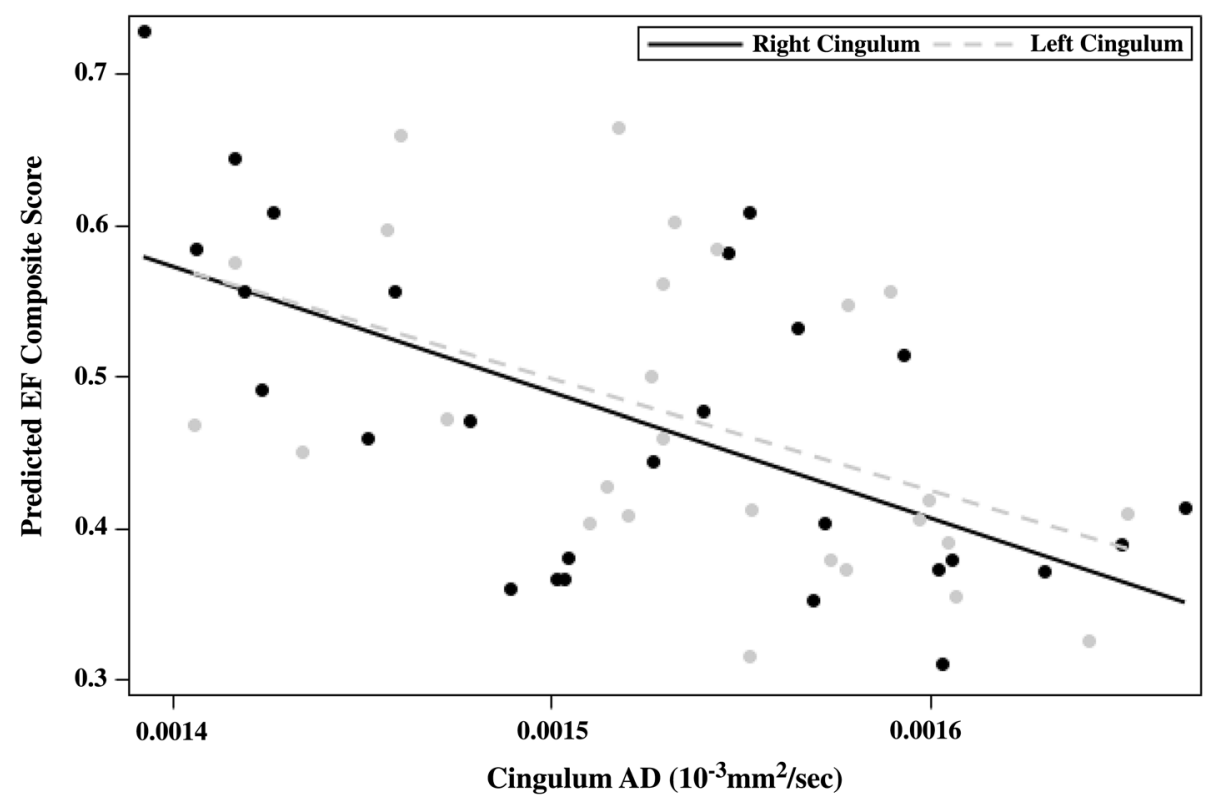

between the left and right cingulum and EF composite performance controlling for all covariates are depicted in Fig. 2. None of the models for any of the other white matter tracts were statistically significant after correction.

\section{Functional regression analyses (FADTTS)}

To confirm initial findings from regression analyses, we used FADTTS to conduct a global curve analysis of AD values along the right cingulum. Consistent with the primary analysis results, the right cingulum $\mathrm{AD}$ curve was significantly associated with EF composite scores $(p=0.034)$. Follow-up analyses of local AD values along the right cingulum indicated which portions of the tract were significantly related to EF (see Fig. 3). After FDR correction for multiple comparisons, associations between the anterior portion of the right cingulum tract and $\mathrm{EF}$ at 3 years of age remained significant. 


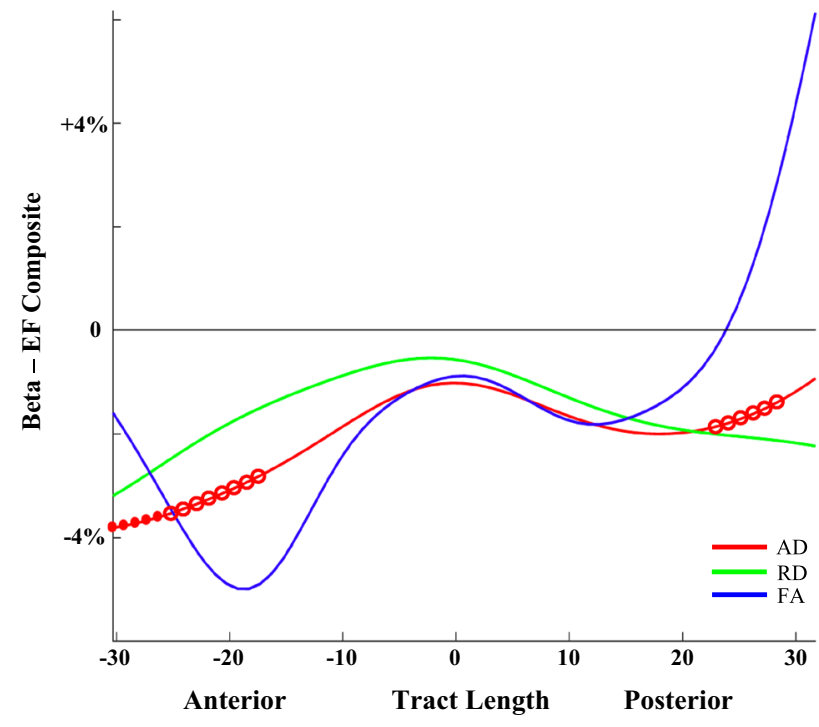

Fig. 3 Neonatal cingulum $\mathrm{AD}$ values are associated with executive function at 3 years of age. Circles represent significant associations along right cingulum, solid circles indicate significance after FDR correction. $A D$ axial diffusivity, $R D$ radial diffusivity, $F A$ fractional anisotropy

\section{Supplementary models}

Although we chose to focus our analyses on the DTI parameter $\mathrm{AD}$, as it is considered the most stable parameter for the less-myelinated neonatal brain, to be consistent with other reports and to ensure the accuracy of this assumption the primary regression analyses were conducted for FA and RD parameters (see Supplementary Tables 1-4). None of these regression models met conventional cutoffs for statistical significance after FDR correction for multiple comparisons.

\section{Discussion}

The goal of this study was to investigate the relation between neonatal white matter microstructure and children's performance on an EF battery at 3 years of age. White matter tracts were selected a priori based on results from previous studies of older children and adults that showed significant associations with EF abilities. One of the most commonly reported tracts to be linked with EF in children and adults is the cingulum. In the current study, we replicated this finding in a much younger sample, showing that despite a small sample size, neonatal $\mathrm{AD}$ values in both the left and right cingulum were significantly associated with children's EF performance 3 years later. After correction for multiple comparisons, the right cingulum remained significantly related to $\mathrm{EF}$ at 3 years of age. This association was not significant for any of the other white matter tracts of interest. An additional, more in-depth analysis (FADTTS) of diffusion properties along the right cingulum supported the initial results from the tract average analysis. To our knowledge, no other studies to date have examined associations between white matter microstructure in neonates and later EF performance.

The significant findings regarding the cingulum are interesting and suggest a potentially important role for this tract in forming the basis for later developing complex cognitive abilities that comprise EF. The cingulum tract and the cingulate cortex region have been shown to be important for attentional skills such as conflict detection and error monitoring (Agam et al. 2011; Melcher et al. 2008). Further, this region is an important contributor to the development of both cognitive and emotional-regulatory abilities. For example, the anterior cingulate cortex is strongly associated with cognitive control, including self-regulatory abilities across childhood, adolescence, and into early adulthood, with this relationship being strongest during early childhood (Fjell et al. 2012). This region has also been studied in infants and preschool-aged children, with EEG studies linking frontal activity to both concurrent and future performance tasks of EF behaviors (Cuevas et al. 2012; Swingler et al. 2011; Whedon et al. 2016). The cingulate cortex, including the cingulum, is recognized as a central component of the executive attention network, which is integral for the development of higher-order cognitive abilities including EF (Conejero and Rueda 2017; Petersen and Posner 2012; Posner and Petersen 1990). The executive attention network as a whole is not considered to be fully functional until later in childhood, as it includes projections to the later-developing prefrontal cortex. However, the functionality of the cingulate region, an important component of the executive attention network, also likely supports the emergence of EF.

Our findings suggest that structural properties of the cingulum shortly after birth may have predictive utility for emerging EF. Although the other tracts examined in this study (i.e., SLF, ARC, and UNC) have been shown to support later EF abilities, one explanation for the differential pattern in our findings may have to do with the order and rate of maturation of individual tracts. Using a variety of analytic techniques, researchers have explored the relative maturity of major white matter fiber bundles in early infancy. Most of this research has established that the cingulum is clearly identifiable and relatively mature at birth compared to other association tracts (Hermoye et al. 2006; Huang et al. 2006). However, the cingulum is also one of the tracts that takes the longest to mature (Dubois et al. 2008), with peak maturation occurring in the 20s (Lebel et al. 2008). DTI parameters provide unique information about the changing microstructural properties of white matter tracts. Of these parameters, previous research suggests that compared to $\mathrm{FA}, \mathrm{AD}$ and $\mathrm{RD}$ may be more reliable measures of early structural integrity. Further, early in life, AD may be a better indicator of later cognitive development as it is thought to represent white 
matter organization and integrity as opposed to myelination (Lee et al. 2017). Our results are in line with this notion, as neonatal $\mathrm{AD}$ values (but not FA or $\mathrm{RD}$ ) were significantly associated with EF 3 years later.

Though the main findings in this study are intriguing, several study limitations should be considered. First, the sample size was small and consisted of a convenience sample of children who had successfully completed a neonatal MRI scan and who were 3 years old at the time of EF data collection. Thus, this study was underpowered to detect anything except moderate to large effects. Caution should be used in interpreting results given the increased chance of Type II errors; the effect sizes for many of the white matter tracts may have been statistically significant with a larger sample size. Relatedly, many researchers have reported strong associations between gestational age at birth and EF (Hodel et al. 2017; Mulder et al. 2009), but we did not find this pattern in our sample. This lack of an effect may also be due to the small sample size. Second, general measures of cognitive development, such as intelligence or language development, were not collected. This limitation undermined the possibility of making inferences about the unique contributions of white matter tracts to EF above and beyond general cognitive ability. However, our inclusion of children's simple reaction time as a covariate allowed us to detect relationships between DTI measures and EF that were independent of simple processing speed. Third, there was variability in regards to the chronological age at which neonates were scanned. As described in the Methods, this was due to an adjusted visit schedule designed to account for differences in gestational term length. Lastly, while the white matter tracts selected for analysis in this study have been shown to be largely established at the time of birth, it is important to note that major changes in fiber density, myelination, and neural connectivity take place in early childhood and continue across the life span (Lamantia and Rakic 1994; Lebel et al. 2008).

Although the limitations of this study preclude any broad conclusions about associations between white matter microstructure at birth and emerging $\mathrm{EF}$, the results suggest a promising direction for future research. Results are consistent with previous research highlighting the cingulum as important for EF abilities, suggesting that our findings are likely to be replicated with a larger sample. These findings prompt a number of questions for future research, such as a consideration of the individual trajectories of white matter microstructural development from birth into early childhood and how these trajectories relate to emerging EF abilities. Examining these associations would greatly expand our understanding of early brain-behavior interactions.

\section{Conclusions}

This study suggests that individual differences in neonatal white matter are related to EF in early childhood. EF plays an important role in social and academic aspects of school readiness, which has resulted in numerous efforts to enhance EF in early childhood, especially for children who are at increased risk for academic difficulties. Our current understanding of brain-behavior relationships very early in life is limited. These results raise the prospect that individual differences in neonatal brain development and structural measures of early white matter may be informative of later cognitive function. The utility of such information could ultimately serve to identify children at risk for EF deficits and guide intervention efforts early in life.

Acknowledgements The collection and processing of neuroimaging data for this project were supported with Grants from National Institutes of Mental Health: K01MH099411 (Short, PI) and MH064065, MH 070890 (Gilmore, PI), and the National Institute of Child Health and Human Development HD053000 (Gilmore, PI), HD079124. The collection of executive function data for this project was supported by Institute of Educational Sciences grant R324A120033 (Willoughby, PI). Dr. Stephens was supported by the National Institutes of Mental Health training Grant T32-MH10644. The views expressed in this manuscript are those of the authors and do not necessarily represent the opinions or position of listed funding agencies.

\section{Compliance with ethical standards}

Conflict of interest The authors declare that they have no conflict of interest.

Ethical approval All procedures performed in studies involving human participants were in accordance with the ethical standards of the institutional and/or national research committee and with the 1964 Helsinki Declaration and its later amendments or comparable ethical standards.

Informed consent Informed consent was obtained from all individual participants included in the study.

\section{References}

Agam Y, Hamalainen MS, Lee AKC et al (2011) Multimodal neuroimaging dissociates hemodynamic and electrophysiological correlates of error processing. Proc Natl Acad Sci 108:17556-17561. https://doi.org/10.1073/pnas.1103475108

Ball G, Aljabar P, Zebari S et al (2014) Rich-club organization of the newborn human brain. Proc Natl Acad Sci 111:7456-7461. https ://doi.org/10.1073/pnas.1324118111

Baum GL, Ciric R, Roalf DR et al (2016) Modular segregation of structural brain networks supports the development of executive function in youth. Curr Biol 27:1561-1572.e8. https://doi. org/10.1016/j.cub.2017.04.051

Beaulieu C (2002) The basis of anisotropic water diffusion in the nervous system—a technical review. NMR Biomed 15:435-455. https ://doi.org/10.1002/nbm.782 
Bendlin BB, Fitzgerald ME, Ries ML et al (2010) White matter in aging and cognition: a cross-sectional study of microstructure in adults aged eighteen to eighty-three. Dev Neuropsychol 35:257277. https://doi.org/10.1080/87565641003696775

Bettcher BM, Mungas D, Patel N et al (2016) Neuroanatomical substrates of executive functions: beyond prefrontal structures. Neuropsychologia 85:100-109. https://doi.org/10.1016/j.neuropsych ologia.2016.03.001

Blair C, Diamond A (2008) Biological processes in prevention and intervention: the promotion of self-regulation as a means of preventing school failure. Dev Psychopathol 20:899-911. https://doi. org/10.1017/S0954579408000436

Burzynska AZ, Nagel IE, Preuschhof C et al (2011) Microstructure of frontoparietal connections predicts cortical responsivity and working memory performance. Cereb Cortex 21:2261-2271. https://doi.org/10.1093/cercor/bhq293

Conejero A, Rueda MR (2017) Early development of executive attention. J Child Adolesc Behav 5:341. https://doi. org/10.4172/2375-4494.1000341

Cuevas K, Swingler MM, Bell MA et al (2012) Measures of frontal functioning and the emergence of inhibitory control processes at 10 months of age. Dev Cogn Neurosci 2:235-243. https://doi. org/10.1016/j.den.2012.01.002

Davis EP, Buss C, Muftuler LT et al (2011) Children's brain development benefits from longer gestation. Front Psychol 2:1-7. https ://doi.org/10.3389/fpsyg.2011.00001

Diamond A (2013) Executive functions. Annu Rev Psychol 64:135168. https://doi.org/10.1146/annurev-psych-113011-143750

Dubois J, Dehaene-Lambertz G, Perrin M et al (2008) Asynchrony of the early maturation of white matter bundles in healthy infants: quantitative landmarks revealed noninvasively by diffusion tensor imaging. Hum Brain Mapp 29:14-27. https://doi. org $/ 10.1002 / \mathrm{hbm} .20363$

Dubois J, Dehaene-Lambertz G, Kulikova S, Poupon C (2014) The early development of brain white matter: a review of imaging studies in fetuses, newborns and infants. Neuroscience 276:4871. https://doi.org/10.1016/j.neuroscience.2013.12.044

Elison JT, Wolff JJ, Heimer DC et al (2013) Frontolimbic neural circuitry at 6 months predicts individual differences in joint attention at 9 months. Dev Sci 16:186-197. https://doi.org/10.1111/ desc. 12015

Fields RD (2010) Change in the brain's white matter. Science (80-) 330:768-769. https://doi.org/10.1126/science.1199139

Filley CM, Fields RD (2016) White matter and cognition: making the connection. J Neurophysiol 116:2093-2104. https://doi. org/10.1152/jn.00221.2016

Fjell AM, Walhovd KB, Brown TT et al (2012) Multimodal imaging of the self-regulating developing brain. Proc Natl Acad Sci 109:19620-19625. https://doi.org/10.1073/pnas.1208243109

Gao W, Zhu H, Giovanello KS et al (2009) Evidence on the emergence of the brain's default network from 2-week-old to 2-yearold healthy pediatric subjects. Proc Natl Acad Sci 106:67906795. https://doi.org/10.1073/pnas.0811221106

Geng X, Gouttard S, Sharma A et al (2012) Quantitative tract-based white matter development from birth to age 2 years. Neuroimage 61:542-557. https://doi.org/10.1016/j.neuroimage.2012.03.057

Gilmore JH, Schmitt JE, Knickmeyer RC et al (2010) Genetic and environmental contributions to neonatal brain structure: a twin study. Hum Brain Mapp 31:1174-1182. https://doi.org/10.1002/ hbm.20926

Goodlett CB, Fletcher PT, Gilmore JH, Gerig G (2009) Group analysis of DTI fiber tract statistics with application to neurodevelopment. Neuroimage 45:S133-S142. https://doi.org/10.1016/j. neuroimage.2008.10.060
Hendry A, Jones EJH, Charman T (2016) Executive function in the first three years of life: precursors, predictors and patterns. Dev Rev 42:1-33

Hermoye L, Saint-Martin C, Cosnard G et al (2006) Pediatric diffusion tensor imaging: normal database and observation of the white matter maturation in early childhood. Neuroimage 29:493-504. https://doi.org/10.1016/j.neuroimage.2005.08.017

Hodel AS, Senich KL, Jokinen C et al (2017) Early executive function differences in infants born moderate-to-late preterm. Early Hum Dev 113:23-30. https://doi.org/10.1016/j.earlhumdev .2017.07.007

Huang H, Zhang J, Wakana S et al (2006) White and gray matter development in human fetal, newborn and pediatric brains. Neuroimage 33:27-38. https://doi.org/10.1016/j.neuroimage.2006.06.009

Kantarci K, Senjem ML, Avula R et al (2011) Diffusion tensor imaging and cognitive function in older adults with no dementia. Neurology 77:26-34. https://doi.org/10.1212/WNL.0b013e31822313dc

Kerchner GA, Racine CA, Hale S et al (2012) Cognitive processing speed in older adults: relationship with white matter integrity. PLoS One 7:e50425. https://doi.org/10.1371/journal.pone.00504 25

Keunen K, Counsell SJ, Benders MJNL (2017) The emergence of functional architecture during early brain development. Neuroimage 160:2-14. https://doi.org/10.1016/j.neuroimage.2017.01.047

Knickmeyer RC, Gouttard S, Kang C et al (2008) A structural MRI study of human brain development from birth to 2 years. J Neurosci 28:12176-12182. https://doi.org/10.1523/JNEUR OSCI.3479-08.2008

Knickmeyer RC, Xia K, Lu Z et al (2016) Impact of demographic and obstetric factors on infant brain volumes: a population neuroscience study. Cereb Cortex 27:5616-5625. https://doi.org/10.1093/ cercor/bhw331

Lamantia AS, Rakic P (1994) Axon overproduction and elimination in the anterior commissure of the developing rhesus monkey. J Comp Neurol 340:328-336. https://doi.org/10.1002/cne.903400304

Lebel C, Walker L, Leemans A et al (2008) Microstructural maturation of the human brain from childhood to adulthood. Neuroimage 40:1044-1055. https://doi.org/10.1016/j.neuroimage.2007.12.053

Lebel C, Warner T, Colby J et al (2013) White matter microstructure abnormalities and executive function in adolescents with prenatal cocaine exposure. Psychiatry Res Neuroimaging 213:161-168. https://doi.org/10.1016/j.pscychresns.2013.04.002

Lee SJ, Steiner RJ, Yu Y et al (2017) Common and heritable components of white matter microstructure predict cognitive function at 1 and 2 years. Proc Natl Acad Sci 114:148-153. https://doi. org/10.1073/pnas.1604658114

Liu Z, Wang Y, Gerig G et al (2010) Quality control of diffusion weighted images. Proc SPIE Int Soc Opt Eng. https://doi. org/10.1117/12.844748

Melcher T, Falkai P, Gruber O (2008) Functional brain abnormalities in psychiatric disorders: neural mechanisms to detect and resolve cognitive conflict and interference. Brain Res Rev 59:96-124. https://doi.org/10.1016/j.brainresrev.2008.06.003

Miller SE, Marcovitch S (2015) Examining executive function in the second year of life: coherence, stability, and relations to joint attention and language. Dev Psychol 51:101-114. https://doi. org/10.1037/a0038359

Mulder H, Pitchford NJ, Hagger MS, Marlow N (2009) Development of executive function and attention in preterm children: a systematic review. Dev Neuropsychol 34:393-421. https://doi. org/10.1080/87565640902964524

Mundy P, Jarrold W (2010) Infant joint attention, neural networks and social cognition. Neural Netw 23:985-997. https://doi. org/10.1016/j.neunet.2010.08.009 
Mundy P, Newell L (2007) Attention, joint attention, and social cognition. Curr Drections Psychol Sci 16:269-274. https://doi.org/10.1 111/j.1467-8721.2007.00518.x

Nagy Z, Westerberg H, Klingberg T (2004) Maturation of white matter is associated with the development of cognitive functions during childhood. J Cogn Neurosci 16:1227-1233. https://doi. org/10.1162/0898929041920441

Oguz I, Farzinfar M, Matsui J et al (2014) DTIPrep: quality control of diffusion-weighted images. Front Neuroinform 8:4. https://doi. org/10.3389/fninf.2014.00004

Ouyang M, Dubois J, Yu Q, Mukherjee P, Huang H (2019) Delineation of early brain development from fetuses to infants with diffusion MRI and beyond. Neuroimage 185:836-850. https://doi. org/10.1016/j.neuroimage.2018.04.017

Perry ME, McDonald CR, Hagler DJ et al (2009) White matter tracts associated with set-shifting in healthy aging. Neuropsychologia 47:2835-2842. https://doi.org/10.1016/j.neuropsychologi a.2009.06.008

Peters BD, Ikuta T, DeRosse P et al (2014) Age-related differences in white matter tract microstructure are associated with cognitive performance from childhood to adulthood. Biol Psychiatry 75:248-256. https://doi.org/10.1016/j.biopsych.2013.05.020

Petersen SE, Posner MI (2012) The attention system of the human brain: 20 years after. Annu Rev Neurosci 35:73-89. https://doi. org/10.1146/annurev-neuro-062111-150525

Posner MI, Petersen SE (1990) The attention system of the human brain. Annu Rev Neurosci 13:25-42. https://doi.org/10.1146/ annurev.ne.13.030190.000325

Rasmussen JM, Kruggel F, Gilmore JH et al (2017) A novel maturation index based on neonatal diffusion tensor imaging reflects typical perinatal white matter development in humans. Int J Dev Neurosci 56:42-51. https://doi.org/10.1016/j.ijdevneu.2016.12.004

Sasson E, Doniger GM, Pasternak O et al (2013) White matter correlates of cognitive domains in normal aging with diffusion tensor imaging. Front Neurosci 7:1-13. https://doi.org/10.3389/fnins 2013.00032

Seghete KLM, Herting MM, Nagel BJ (2013) White matter microstructure correlates of inhibition and task-switching in adolescents. Brain Res 1527:15-28. https://doi.org/10.1016/j.brain res.2013.06.003

Short SJ, Elison JT, Goldman BD et al (2013) Associations between white matter microstructure and infants' working memory. Neuroimage 64:156-166. https://doi.org/10.1016/j.neuroimage .2012.09.021

Smith SM (2002) Fast robust automated brain extraction. Hum Brain Mapp 17:143-155. https://doi.org/10.1002/hbm.10062

Swingler MM, Willoughby MT, Calkins SD (2011) EEG power and coherence during preschoolers' performance of an executive function battery. Dev Psychobiol 53:771-784. https://doi.org/10.1002/ dev.20588

Urger SE, De Bellis MD, Hooper SR et al (2015) The superior longitudinal fasciculus in typically developing children and adolescents: diffusion tensor imaging and neuropsychological correlates. J Child Neurol 30:9-20. https://doi.org/10.1177/088307381352050 3
Vaughan Van Hecke A, Mundy P, Block JJ et al (2011) Infant responding to joint attention, executive processes, and self-regulation in preschool children. Infant Behav Dev 35:303-311. https://doi. org/10.1016/j.infbeh.2011.12.001

Verde AR, Budin F, Berger J-B et al (2014) UNC-Utah NA-MIC framework for DTI fiber tract analysis. Front Neuroinform 7:51. https:// doi.org/10.3389/fninf.2013.00051

Vestergaard M, Skakmadsen K, Baare W et al (2011) White matter microstructure in superior longitudinal fasciculus associated with spatial working memory performance in children. J Cogn Neurosci 23:2135-2146. https://doi.org/10.1162/jocn.2010.21592

Wang Y, Gupta A, Liu Z et al (2011) DTI registration in atlas based fiber analysis of infantile Krabbe disease. Neuroimage 55:15771586. https://doi.org/10.1016/j.neuroimage.2011.01.038

Whedon M, Perry NB, Calkins SD, Bell MA (2016) Changes in frontal EEG coherence across infancy predict cognitive abilities at age 3: the mediating role of attentional control. Dev Psychol 52:13411352. https://doi.org/10.1037/dev0000149

Willoughby M, Blair C (2011) Test-retest reliability of a new executive function battery for use in early childhood. Child Neuropsychol 17:564-579. https://doi.org/10.1080/09297049.2011.554390

Willoughby MT, Blair CB (2016) Measuring executive function in early childhood: a case for formative measurement. Psychol Assess 28:319-330. https://doi.org/10.1037/pas0000152

Willoughby MT, Blair C, Wirth RJ, Greenberg M (2010) The measurement of executive function at age 3 years: psychometric properties and criterion validity of a new battery of tasks. Psychol Assess 22:306-317. https://doi.org/10.1037/a0018708

Willoughby MT, Wirth RJ, Blair C (2012) Executive function in early childhood: longitudinal measurement invariance and developmental change. Psychol Assess 24:418-431. https://doi.org/10.1037/ a0025779

Willoughby MT, Pek J, Blair CB (2013) Measuring executive function in early childhood: a focus on maximal reliability and the derivation of short forms. Psychol Assess 25:664-670. https:// doi.org/10.1037/a0031747

Willoughby MT, Magnus B, Vernon-Feagans L et al (2016) Developmental delays in executive function from 3 to 5 years of age predict kindergarten academic readiness. J Learn Disabil 50:359_ 372. https://doi.org/10.1177/0022219415619754

Yap PT, Fan Y, Chen Y et al (2011) Development trends of white matter connectivity in the first years of life. PLoS One 6:e24678. https ://doi.org/10.1371/journal.pone.0024678

Zatorre RJ, Fields RD, Johansen-Berg H (2012) Plasticity in gray and white: neuroimaging changes in brain structure during learning. Nat Neurosci 15:528-536. https://doi.org/10.1038/nn.3045

Zhu H, Kong L, Li R et al (2011) FADTTS: functional analysis of diffusion tensor tract statistics. Neuroimage 56:1412-1425. https:// doi.org/10.1016/j.neuroimage.2011.01.075

Publisher's Note Springer Nature remains neutral with regard to jurisdictional claims in published maps and institutional affiliations. 